

\title{
New results in the volatile odorous compounds of French cheeses
}

\author{
A Gallois, David Langlois
}

\section{To cite this version:}

A Gallois, David Langlois. New results in the volatile odorous compounds of French cheeses. Le Lait, 1990, 70 (2), pp.89-106. hal-00929199

\section{HAL Id: hal-00929199 \\ https://hal.science/hal-00929199}

Submitted on 1 Jan 1990

HAL is a multi-disciplinary open access archive for the deposit and dissemination of scientific research documents, whether they are published or not. The documents may come from teaching and research institutions in France or abroad, or from public or private research centers.
L'archive ouverte pluridisciplinaire HAL, est destinée au dépôt et à la diffusion de documents scientifiques de niveau recherche, publiés ou non, émanant des établissements d'enseignement et de recherche français ou étrangers, des laboratoires publics ou privés. 


\title{
New results in the volatile odorous compounds of French cheeses
}

\author{
A Gallois *, D Langlois \\ INRA, Laboratoire de recherches sur les arômes, BV 1540, 21034 Dijon Cedex, France
}

(Received 7 December 1988; accepted 10 April 1989)

\begin{abstract}
Summary - The volatile odorous constituents of five French blue cheeses (three Roquefort, a Bleu des Causses and a Bleu d'Auvergne) were isolated by high vacuum distillation, separated by gas chromatographic analysis and subjected to mass spectral analysis. One hundred and twenty nine compounds were identified and quantified. Methyl ketones were the majors compounds for all cheeses and represented 50 to $75 \%$ of the total odorous profile. Secondary alcohols, esters were abundant, especially in the Roquefort samples. The Bleu des Causses was rich in sulfur compounds. Lactones, primary alcohols and aldehydes were more abundant in the Bleu d'Auvergne.
\end{abstract}

\section{Roquefort - Bleu des Causses - Bleu d'Auvergne - volatile compound - flavour}

Résumé - Nouveaux développements dans l'étude des composés volatils odorants des fromages français à pâte persillée. Les composés volatils odorants de 5 fromages à pâte persillée (3 Roqueforts fabriqués avec des souches de Penicillium roqueforti différentes, 1 Bleu des Causses et 1 Bleu d'Auvergne) ont été extraits par distillation sous vide poussé, séparés par chromatographie en phase gazeuse et analysés par spectrométrie de masse. Cent vingt neuf composés ont été identifiés et quantifiés. Les méthyl-cétones étaient majoritaires dans tous les fromages et représentaient quantitativement 50 à $75 \%$ du profil odorant total. Les alcools secondaires et les esters étaient abondants, surtout dans les échantillons de roquefort. Le Bleu des Causses était le plus riche en composés soufrés et le bleu d'Auvergne le plus riche en lactones, alcools primaires et aldéhydes.

roquefort - bleu des Causses - bleu d'Auvergne - composésvolatil - arôme

\footnotetext{
* Present address: INRA, Laboratoire science de l'aliment, 1, avenue des Olympiades, 91305 Massy, France
} 


\section{INTRODUCTION}

The homologous series of odd-carbon chain methyl ketones have been identified and characterized as the main compounds responsible for the unique flavour of blue cheeses (Anderson, Day, 1966). Most of the studies carried out in the last 20 years have been restricted to the quantitative determination of carbonyl compounds in various blue varieties : American Blue (Schwartz, Parks, 1963), Roquefort (Schwartz et al, 1963), Normanna cheese (Norwegian Blue : Svensen, Ottestad, 1969), German Edelpilz (Ney, Wirotama, 1972) and more recently, Stilton (Madkor et al, 1987).

The typical blue- or Roquefort-type odour has been found to persist after removal of all monocarbonyls (Schwartz, Parks, 1963). But neutral non-carbonyl volatile compounds of blue cheese have not been frequently studied. No quantitative data have been published. Only several esters and lactones have been reported in blue cheese (Day, Anderson, 1965; Ney, Wirotoma, 1972; Jolly, Kosikowski, 1975).
This paper reports the quantitative analysis of the neutral volatile compounds of different French blue cheeses. Three samples of Roquefort (produced from ewe's milk), a Bleu des Causses and a Bleu d'Auvergne (both produced from cow's milk) were analyzed. It was of interest to determine to what extent the organoleptic differences observed between these cheeses could be explained by the neutral volatiles occurring in the profiles.

\section{MATERIAL AND METHODS}

\section{Cheese samples}

Three Roquefort samples manufactured under commercial conditions with 3 different Penicillium roqueforti strains: PF, PO and PG (Table I), were obtained after $210 \mathrm{~d}$ of ripening from the Etablissements du Groupe Société des Caves de Roquefort (Roquefort, France). A Bleu des Causses sample manufactured with the same PG strain was obtained after $100 \mathrm{~d}$ of ripening from Valmont (Rodez, France). Except for the milk origin, the manufacturing procedures were similar for both Roquefort and Bleu des Causs-

Table I. Properties of the 3 strains of Penicillium roqueforti used in the manufacture of the Roquefort samples (by courtesy of M Assenat, Roquefort, France)

Propriétés des souches de Penicillium roqueforti utilisées pour la fabrication des échantillons de Roquefort (avec la gracieuse permission de M Assenat, Roquefort, France)

\begin{tabular}{|c|c|c|c|}
\hline & \multicolumn{3}{|c|}{ Strains } \\
\hline & $P F$ & $P O$ & $P G$ \\
\hline $\begin{array}{l}\text { proteolytic activity } \\
\text { lipolytic activity } \\
\text { mycelium aspect } \\
\text { commercial presentation } \\
\text { organoleptic properties }\end{array}$ & $\begin{array}{l}\text { low } \\
\text { low } \\
\text { dark } \\
\text { good } \\
\text { neutral } \\
\text { flavor }\end{array}$ & $\begin{array}{l}\text { high } \\
\text { rather high } \\
\text { pale } \\
\text { poor } \\
\text { good flavor } \\
\text { very typical }\end{array}$ & $\begin{array}{l}\text { medium } \\
\text { medium } \\
\text { dark } \\
\text { good } \\
\text { good flavor } \\
\text { often pungent }\end{array}$ \\
\hline
\end{tabular}


es, A Bleu d'Auvergne sample was purchased at a local market after a ripening period of approximately $45 \mathrm{~d}$. ied.

All samples were stored at $-20^{\circ} \mathrm{C}$ until stud-

\section{Rapid measure of the total free fatty acids}

$100 \mathrm{~g}$ of cheese, triturated for $15 \mathrm{~min}$ at $30^{\circ} \mathrm{C}$, were centrifugated $(12000 \mathrm{~g})$ for $10 \mathrm{~min}$ at $15^{\circ} \mathrm{C}$. An aliquot $(2 \mathrm{~g})$ of the upper fat layer was withdrawn and diluted in $20 \mathrm{ml}$ of a mixture ethanol : diethyl ether $(1: 1, \mathrm{v} / \mathrm{v})$. After addition of $0.5 \mathrm{ml}$ of $1 \%$ ethanolic phenolphtalein, the solution was titrated with $0.1 \mathrm{~N}$ ethanolic sodium hydroxyde. $20 \mathrm{ml}$ of ethanol : diethyl ether $(1: 1)$ were neutralized under the same conditions to estimate the background titration.

\section{Separation and measurement of sulfur compounds}

The static headspace method, described by Qvist, von Sydow (1976) was used to analyze the volatile sulfur compounds fo the blue cheeses. $50 \mathrm{~g}$ of the deep-frozen cheese were homogenized at $0{ }^{\circ} \mathrm{C}$ with $100 \mathrm{ml}$ of distilled water in a flask which was then rotated in an inclined position in a water bath at $32{ }^{\circ} \mathrm{C}$ to obtain equilibrium.

After $30 \mathrm{~min}, 200 \mathrm{ml}$ of the vapors surrounding the sample were trapped in an injection loop cooled with liquid nitrogen. Heating of the loop allowed the direct injection of the trapped compounds in a Girdel 3000 chromatograph (Delsi) equipped with a Tracor sulfur specific flame photometric detector.

The column used was a $6.4 \mathrm{~m} \times 2 \mathrm{~mm}$ id glass tube packed with $5 \%$ lgepal CO 630 on Chromosorb G. AW DMCS (80/100 Mesh). Oven temperature was programmed from $10^{\circ} \mathrm{C}$ to $110^{\circ} \mathrm{C}$ at $4{ }^{\circ} \mathrm{C} / \mathrm{min}$ after an initial isothermal period of $15 \mathrm{~min}$. The nitrogen carrier gas flow was $20 \mathrm{ml} / \mathrm{min}$.

Compounds were identified according to their retention time (calculated relative to methyl disulfide), and by comparison with the retention times of authentic compounds when available. Concentrations of some sulfur compounds could be calculated using external standards (injected under the same conditions).

\section{Extraction of neutral volatile compounds}

A vacuum degassing (2.10-3 Torr; $5 \mathrm{~h} 30)$ and a cold-finger molecular distillation $\left(3.10^{-5}\right.$ Torr; $4 \mathrm{~h}$ 30) were successively carried out. The apparatus and procedures were similar to those described by Forss \& Holloway (1967). Three extractions were performed for each type of cheese using each $150 \mathrm{~g}$ of ground deep-frozen cheese.

Aqueous distillates, collected in the liquid nitrogen traps and removed from the cold-finger by rinsing with distilled water, were pooled. After adjusting to $\mathrm{pH} 9$ (with sodium hydoxide $2 \mathrm{~N}$ ), the distillates were saturated with sodium chloride and thrice extracted in a separatory funnel with trichlorofluoro-methane (Forane 11, Atochem) : $1 \times 45 \mathrm{ml}$ and $2 \times 20 \mathrm{ml}$. The aqueous was odorless.

After drying over anhydrous sodium sulfate, an internal standard was added to the solvent extracts (amyl 2-methyl butanoate : $10^{-2} \mu l / 150$ $\mathrm{g}$ of cheese ie, $0.053 \mathrm{mg} / \mathrm{kg}$ ), which were then carefully concentrated under reflux to a small volume $(250 \mu \mathrm{l})$.

\section{Gas chromatography analyses}

All separations were performed on a Girdel DI 700 chromatograph (Delsi) equipped with a 30 $\mathrm{m} \times 0.32 \mathrm{~mm}$ id fused silica capillary column (J\&W Scientific Inc.) bonded with DB5 (95\% methyl-, $5 \%$ phenyl-polysiloxane). The film thickness was $1 \mu \mathrm{m}$. The hydrogen carrier gas flow was $50 \mathrm{~cm} / \mathrm{s}$. The oven temperature was programmed from $30-220^{\circ} \mathrm{C}$ at a $2^{\circ} \mathrm{C} / \mathrm{min}$ rate. FID detector and split-splitless injector temperatures were maintained at $240^{\circ} \mathrm{C}$.

Retention indices were determined using these conditions. Peak areas were recorded with an Enica 10 integrator (Delsi). 


\section{Mass spectrometry}

Electron impact mass spectra were recorded using a Nermag R10 - 10 spectrometer (Nermag) coupled with a Girdel 31 chromatograph (Delsi). The fused silica capillary column DB5 (60 $\mathrm{m} \times 0.32 \mathrm{id}$, film thickness $1 \mu \mathrm{m}$ ) was directly connected to the ion source. The operating GC conditions were the same as above. The ion source energy was $70 \mathrm{eV}$. The temperatures of the ion source and the interface were respectively 150 and $2800^{\circ} \mathrm{C}$. The scanning rate was $0.8 \mathrm{~s}$ from 25 to $300 \mathrm{amu}$.

All identifications were made by comparison of the mass spectra with those of reference compounds, when available or with bibliographic data.

\section{RESULTS AND DISCUSSION}

\section{Titratable free acidity}

The free fatty acids (FFA), which are important compounds in the flavour of blue cheese (Day, 1967), were much more abundant in Bleu des Causses than in all other cheeses (table II). They were twice as abundant in Roquefort PG than in the 2 other Roquefort samples. It has been reported that FFA accumulated during ripening to relatively greater proportions in blue cheese than in most other types of cheese
(Woo et al, 1984). In order to estimate the extent of lipolysis during ripening, it would have been interesting to know the initial values of total FFA in the milk.

\section{Sulfur compounds}

Despite the relative high standard deviations, large variations in concentrations of sulfur compounds between cheeses were obvious (table III). The concentrations of hydrogen sulfide and methanetiol were not calculated because calibration curves of these highly volatile compounds were not established.

Sulfur compounds were much more abundant in Bleu des Causses (especially methyl sulfide and methyl disulfide). Nevertheless, hydrogen sulfide, which is generally reported in all types of matured cheeses, was not found in Bleu des Causses. Similar amounts of hydrogen sulfide and dimethyl sulfide were found in the 3 Roquefort cheeses. Methanethiol and dimethyl disulfide were more abundant in Roquefort PO and $P G$ than in PF, which contained the most important amount of carbon disulfide. However, carbon disulfide was found here at very low levels ( 1 to $5 \mathrm{ppb}$ ) and probably did not contribute to the flavor of blue cheese.

Table II. Free fatty acids contained in the fat fraction of the cheeses expressed in meq $/ 100 \mathrm{~g}$ of fat. Teneur en acides gras libres des fractions lipidiques de chaque fromage (exprimée en meq/100 $\mathrm{g}$ de matière grasse).

a : PF, PO, PG ; Roquefort: CAU : Bleu des Causses; AUV : Bleu d'Auvergne.

Cheeses a
PF
PO
$P G$
$C A U$
AUV

\begin{tabular}{llllll}
\hline FFA & 4.70 & 4.65 & 10.25 & 17.45 & 4.70 \\
\hline
\end{tabular}


Table III. Sulfur compounds found in the headspace of the different cheeses, a : PO, PF, PG : Roquefort; CAU : Bleu des Causses; AUV : Bleu d'Auvergne; ${ }^{b}$ : peak height (average of triplicate analysis); c : concentrations $\left(\mu \mathrm{g} / \mathrm{kg}\right.$ of cheese); calculated whenever reference compounds were available; ${ }^{d}$ : standard deviation of the 3 replicates.

Composés soufrés présents dans l'espace de tête des différents fromages. a : PO, PF, PG : Roquefort; CAU : Bleu des Causses; AUV : Auvergne Blue Cheese; $b$ : hauteur de pic (moyenne de trois répétitions); $c$ : concentrations ( $\mu \mathrm{g} / \mathrm{kg}$ de fromage) calculées chaque fois que les composés de référence étaient disponibles; ${ }^{d}$ : coefficient de variation des trois répétitions.

\begin{tabular}{|c|c|c|c|c|c|c|c|c|c|c|c|c|c|c|c|}
\hline \multirow{2}{*}{$\begin{array}{l}\text { Sulfur } \\
\text { Compounds }\end{array}$} & \multicolumn{3}{|c|}{$P F a$} & \multicolumn{3}{|c|}{$P O^{a}$} & \multicolumn{3}{|c|}{$P G a$} & \multicolumn{3}{|c|}{$C A U^{a}$} & \multicolumn{3}{|c|}{ AUVa } \\
\hline & $H^{b}$ & $c^{c}$ & $\%^{d}$ & $H$ & c & $\%$ & H & c & $\%$ & $H$ & $c$ & $\%$ & $H$ & C & $\%$ \\
\hline $\mathrm{H}_{2} \mathrm{~S}$ & 439 & & 38 & 536 & & 70 & 243 & & 17 & - & & & - & & \\
\hline $\mathrm{CH}_{3} \mathrm{SH}$ & & & 25 & 2216 & & 34 & 1601 & & 32 & 45380 & & 18 & - & & \\
\hline $\mathrm{CH}_{3} \mathrm{SCH}_{3}$ & 1267 & 7 & 20 & 970 & 7 & 42 & 2218 & 11 & 30 & 58345 & 70 & 14 & 256 & 8 & 12 \\
\hline $\mathrm{CS}_{2}$ & 3088 & 5 & 20 & 634 & 3 & 39 & 560 & 2 & 11 & - & & & 95 & 1 & 6 \\
\hline unknown & 164 & & 47 & 120 & & 27 & 512 & & 31 & - & & & 69 & & 41 \\
\hline $\mathrm{CH}_{3} \mathrm{~S}_{2} \mathrm{CH}_{3}$ & 155 & tr & 20 & 7702 & 29 & 27 & 8004 & 29 & 9 & 75985 & 90 & 25 & 1819 & 24 & 25 \\
\hline
\end{tabular}

The detection threshold of dimethyl sulfide was $0.3 \mathrm{ppb}$ in water and $9 \mathrm{ppb}$ in milk and milk fat (Shankaranarayana et al, 1974). Even if the thereshold was not measured in cheese, we supposed that dimethyl sulfide was present in Bleu des Causses at a greater than threshold level (70 ppb), and contributed to the overall aroma of this cheese. In the same way dimethyl disulfide (detection threshold : 3 $\mathrm{ppb}$ in water, Shankaranarayana et al, 1974) could likely be detected in all samples (except perhaps in Roquefort PG).

Sulfur compounds have not been often reported in the studies of blue cheese aroma. Only methanethiol and hydrogen sulfide have been tentatively identified (Anderson, 1966).

\section{Neutral volatile compounds}

\section{General considerations}

One hundred and fifty-two compounds were observed in the Forane 11 extracts of the 5 cheese samples. Eighty-one of them were identified and 48 tentatively identified (table IV). The "apparent" concentrations are given in table IV : the aera ratio of each compound versus the internal standard was calculated without taking into account extraction recoveries or response coefficients. Estimations of the real concentrations were obtained for the most abundant compounds by adding standards to the Bleu d'Auvergne prior to distillation. The ranges of recoveries were $70-90 \%$ for methyl ketones, $50-70 \%$ for both primary and secondary alcohols, $50 \%$ for alkanals, $60 \%$ for lactones and $80-90 \%$ for esters, which were in agreement with the previous results of Forss, Holloway (1967) and Stark et al (1973).

The average standard deviation was $16.6 \%$. The highest values were obtained for the less abundant compounds (below $50 \mu \mathrm{g} / \mathrm{kg}$ ). This could have been foreseen, owing to the heterogeneity of the samples (already noted by Svensen, Ottestad, 1969) and to the extraction method used. However, variations between samples 
Table IV. Volatile compounds of blue cheeses; a : concentration ( $\mu \mathrm{g} / \mathrm{kg}$ of cheese) - average of triplicate analysis; b : standard deviation of the 3 replicates; ${ }^{c}$ : retention indices determined on DB5 column; ${ }^{*}$ : retention indices identical with those of authentic compounds

Composés volatils présents dans les bleus; ${ }^{a}$ : concentration $\left(\mu \mathrm{g} / \mathrm{kg}\right.$ de fromage - moyenne de trois répétitions; ${ }^{b}$ : coefficient de variation des trois répétitions; ${ }^{c}$ : indices de rétention déterminés sur $D B 5 ;{ }^{*}$ : indices de rétention identiques à ceux des composés de référence.

\begin{tabular}{|c|c|c|c|c|c|c|c|c|c|c|c|}
\hline \multirow{3}{*}{ Compounds } & \multicolumn{10}{|c|}{ Blue cheeses } & \multirow[b]{3}{*}{$I R c$} \\
\hline & \multicolumn{2}{|c|}{$P F$} & \multicolumn{2}{|c|}{$P O$} & \multicolumn{2}{|c|}{$P G$} & \multicolumn{2}{|c|}{$C A U$} & \multicolumn{2}{|c|}{$A U V$} & \\
\hline & $\mathrm{Ca}$ & $\%^{b}$ & $c$ & $\%$ & $c$ & $\%$ & $c$ & $\%$ & $c$ & $\%$ & \\
\hline \multicolumn{12}{|l|}{ KETONES } \\
\hline 2-butanone & - & & - & & $\operatorname{tr}$ & & - & & - & & 600 \\
\hline 2-pentanone & 1680 & 2 & 565 & 6 & 9430 & 8 & 3440 & 17 & 340 & 10 & *686 \\
\hline 3-hydroxy 2-butanone & - & & - & & 80 & 12 & - & & - & & *702 \\
\hline 3-methyl 2-pentanone & 10 & 18 & 10 & 9 & 5 & 24 & - & & - & & $* 748$ \\
\hline 2-hexanone & 40 & 7 & 30 & 33 & 145 & 5 & 60 & 12 & 10 & 4 & *790 \\
\hline 5-hepten 2-one & - & & - & & 30 & 6 & 5 & 66 & 10 & 14 & 875 \\
\hline 2-heptanone & 3955 & 6 & 4320 & 32 & 13090 & 18 & 2855 & 8 & 1810 & 2 & *897 \\
\hline 3-methyl 2-heptanone & 5 & 10 & 15 & 4 & 40 & 31 & 5 & 31 & - & & 940 \\
\hline 5 hepten 6-methyl/ 2-one & - & & 100 & 18 & - & & - & & 40 & 4 & 990 \\
\hline 3-octanone & 65 & 4 & - & & 15 & 2 & 10 & 25 & - & & *990 \\
\hline 2-octanone & 55 & 8 & 100 & 6 & 210 & $\overline{5}$ & 40 & 8 & 60 & 2 & *993 \\
\hline 1-Me 1-cyclohexen 3-one & - & & - & & 20 & 10 & - & & - & & 1066 \\
\hline acetophenone & 10 & 18 & 15 & 25 & - & & 15 & 32 & - & & *1 076 \\
\hline 8-nonen 2-one & 380 & 14 & 340 & 25 & 440 & 16 & 340 & 11 & 610 & 13 & 1092 \\
\hline 2-nonanone & 3915 & 10 & 7235 & 10 & 10040 & 11 & 1600 & 6 & 4570 & 18 & $* 1100$ \\
\hline 2-decanone & 370 & 5 & 460 & 8 & 60 & 3 & - & & 60 & 3 & ${ }^{*} 1190$ \\
\hline unsatured ketone & - & & - & & 10 & 22 & - & & - & & 1200 \\
\hline 10-undecen 2-one & 15 & 30 & 35 & 4 & 40 & 14 & 20 & 17 & - & & 1288 \\
\hline 6-undecen 2-one & - & & - & & - & & 40 & 10 & 20 & 19 & 1290 \\
\hline 2-undecanone & 515 & 5 & 990 & 2 & 1090 & 9 & 455 & 14 & 1490 & 10 & *1296 \\
\hline tridecenone & - & & - & & - & & 55 & 30 & 220 & 4 & 1495 \\
\hline
\end{tabular}




\begin{tabular}{|c|c|c|c|c|c|c|c|c|c|c|c|}
\hline \multirow{3}{*}{ Compounds } & \multicolumn{10}{|c|}{ Blue cheeses } & \multirow[b]{3}{*}{$I^{c}$} \\
\hline & \multicolumn{2}{|c|}{$P F$} & \multicolumn{2}{|c|}{$P O$} & \multicolumn{2}{|c|}{$P G$} & \multicolumn{2}{|c|}{$C A U$} & \multicolumn{2}{|c|}{ AUV } & \\
\hline & $C^{a}$ & $\%^{b}$ & $C$ & $\%$ & C & $\%$ & $c$ & $\%$ & $c$ & $\%$ & \\
\hline \multicolumn{12}{|l|}{ KETONES (cont) } \\
\hline $\begin{array}{l}\text { 2-tridecanone } \\
\text { 1-hydroxy } 1 \text {-methylethyl }\end{array}$ & 70 & 26 & 65 & 7 & 115 & 18 & 230 & 24 & 410 & 7 & *1500 \\
\hline acetophenone & 10 & 43 & 10 & 8 & - & & - & & - & & 1520 \\
\hline pentadecanone & - & & 60 & 23 & 30 & 9 & 175 & 26 & 130 & 15 & $* 1704$ \\
\hline unsaturated ketone $\mathrm{C}_{16}$ & - & & - & & 50 & 7 & - & & - & & 1798 \\
\hline Total concentration & 11095 & & 14350 & & 34940 & & 9345 & & 9780 & 0 & \\
\hline \multicolumn{12}{|l|}{ ALCOHOLS } \\
\hline 2-methyl 1-propanol & 70 & 60 & 85 & 17 & $\operatorname{tr}$ & & 90 & 34 & 280 & 18 & ${ }^{*} 626$ \\
\hline 1-butanol & 10 & 59 & 5 & 21 & - & & - & & - & & ${ }^{*} 660$ \\
\hline 2-pentanol & 600 & 16 & - & & 1410 & 19 & 1410 & 10 & 290 & 3 & ${ }^{*} 700$ \\
\hline 3-methyl 1-butanol & 1030 & 4 & 1460 & 5 & 255 & 7 & 110 & 23 & 3180 & 10 & *732 \\
\hline 2-methyl 1-butanol & - & & - & & - & & - & & 980 & 23 & ${ }^{\star} 738$ \\
\hline 1-pentanol & - & & - & & - & & 745 & 27 & - & & *767 \\
\hline 2-hexanol & - & & - & & 5 & 34 & - & & - & & ${ }^{\star} 816$ \\
\hline 1-hexanol & 50 & 27 & 10 & 7 & - & & - & & 10 & 40 & ${ }^{*} 868$ \\
\hline 2-heptanol & 1325 & 4 & 515 & 22 & 2905 & 17 & 785 & 16 & 830 & 1 & ${ }^{*} 900$ \\
\hline alcohol $C_{7}$ & - & & - & & 5 & 6 & - & & - & & 943 \\
\hline 1-heptanol & - & & - & & - & & - & & 10 & 2 & ${ }^{\star} 968$ \\
\hline 1,5-octadien 3-ol & - & & - & & - & & 5 & 15 & - & & \\
\hline 1-octen 3-ol & 20 & 12 & 35 & 6 & 20 & 6 & 10 & 62 & 20 & 4 & ${ }^{\star} 984$ \\
\hline 2-octanol & - & & - & & 20 & 13 & - & & - & & ${ }^{*} 1010$ \\
\hline alcohol & 10 & 40 & - & & - & & - & & - & & \\
\hline 2-nonanol & 710 & 15 & 980 & 14 & 2920 & 22 & 450 & 12 & 970 & 2 & *11 105 \\
\hline phenyl ethanol & 140 & 21 & 145 & 7 & - & & 130 & 5 & 1400 & 5 & *1116 \\
\hline 1-decen 3-ol & 10 & 14 & 25 & 6 & 15 & 7 & - & & - & & 1180 \\
\hline 2-decanol & 5 & 14 & 5 & 14 & - & & 10 & 37 & 10 & 2 & *1208 \\
\hline 2-undecanol & 45 & 17 & 40 & 12 & 115 & 22 & 50 & 18 & 130 & & $* 1300$ \\
\hline Total concentration & 4025 & & 3305 & & 7670 & & 3795 & & 8110 & & \\
\hline
\end{tabular}




\begin{tabular}{|c|c|c|c|c|c|c|c|c|c|c|c|}
\hline \multirow{3}{*}{ Compounds } & \multicolumn{10}{|c|}{ Blue cheeses } & \multirow[b]{3}{*}{$I R^{c}$} \\
\hline & \multicolumn{2}{|c|}{$P F$} & \multicolumn{2}{|c|}{ PO } & \multicolumn{2}{|c|}{$P G$} & \multicolumn{2}{|c|}{$C A U$} & \multicolumn{2}{|c|}{$A U V$} & \\
\hline & $C^{a}$ & $\%^{b}$ & $C$ & $\%$ & $c$ & $\%$ & $c$ & $\%$ & ? & $\%$ & \\
\hline \multicolumn{12}{|l|}{ PHENOLS } \\
\hline 2-methyl 5-ethyl phenol & 5 & 36 & 10 & 24 & 110 & 23 & - & & - & & 1115 \\
\hline 4-ethyl phenol & 30 & 6 & - & & 20 & 11 & $\operatorname{tr}$ & & 10 & 18 & *1167 \\
\hline 3-propyl phenol & 5 & 5 & - & & - & & - & & - & & 1273 \\
\hline Total concentration & 40 & & 10 & & 130 & & $\operatorname{tr}$ & & 10 & & \\
\hline \multicolumn{12}{|l|}{ ESTERS } \\
\hline methyl butanoate & - & & 15 & 4 & 20 & 14 & 25 & 25 & 20 & 5 & *720 \\
\hline methyl isovalerate & - & & - & & - & & 5 & 29 & - & & \\
\hline ethyl butanoate & 50 & 8 & 15 & 22 & 60 & 8 & 55 & 14 & 30 & 14 & *803 \\
\hline isopropyl butanoate & 5 & 70 & 5 & 21 & 10 & 17 & 10 & 22 & - & & *824 \\
\hline sec-amyl acetate & - & & - & & - & & 5 & 32 & 20 & 6 & \\
\hline ethyl isolaverate & - & & - & & - & & 5 & 29 & - & & \\
\hline$n$-amyl acetate & - & & - & & - & & 10 & 2 & - & & \\
\hline methyl hexanoate & 20 & 27 & 90 & 13 & 80 & 3 & 70 & 22 & 70 & 1 & *928 \\
\hline methylthio isolaverate & - & & - & & - & & 5 & 2 & - & & \\
\hline isobutyl butanoate & 5 & 20 & 30 & 34 & 20 & 28 & 10 & 21 & 30 & 3 & *959 \\
\hline butyl butanoate & 30 & 38 & 30 & 27 & - & & - & & - & & *1 002 \\
\hline ethyl hexanoate & 75 & 13 & 70 & 10 & 75 & 12 & 70 & 9 & 60 & 9 & *1 005 \\
\hline 1-Me butyl isobutanoate & - & & - & & 50 & 5 & 15 & 19 & - & & 1053 \\
\hline isopropyl hexanoate & 5 & 59 & 5 & 0 & 60 & 19 & 20 & 9 & - & & *1 056 \\
\hline isoamyl isobutanoate & - & & - & & 170 & 10 & 85 & 26 & - & & 1063 \\
\hline heptyl acetate & - & & - & & - & & - & & 440 & 1 & *1 075 \\
\hline methyl octanoate & 40 & 14 & 225 & 5 & 195 & 21 & 70 & 22 & 60 & 19 & ${ }^{*} 1124$ \\
\hline $\begin{array}{l}\text { Internal standard } \\
\text { isobutyl hexanoate }\end{array}$ & & 1 & 30 & 18 & 40 & 13 & 5 & 30 & 20 & 7 & $*_{1} 150$ \\
\hline ethyl octanoate & 110 & 6 & - & & 110 & 13 & 80 & 7 & 50 & 9 & *1196 \\
\hline 1-Me hexyl butanoate & 10 & 19 & 20 & 5 & 130 & 15 & 15 & 16 & 10 & 30 & *1 214 \\
\hline
\end{tabular}




\begin{tabular}{|c|c|c|c|c|c|c|c|c|c|c|c|}
\hline \multirow{3}{*}{ Compounds } & \multicolumn{10}{|c|}{ Blue cheeses } & \multirow[b]{3}{*}{$I R^{c}$} \\
\hline & \multicolumn{2}{|c|}{$P F$} & \multicolumn{2}{|c|}{$P O$} & \multicolumn{2}{|c|}{$P G$} & \multicolumn{2}{|c|}{$C A U$} & \multicolumn{2}{|c|}{$A U V$} & \\
\hline & $\mathrm{Ca}^{a}$ & $\%^{b}$ & $C$ & $\%$ & $C$ & $\%$ & $c$ & $\%$ & $C$ & $\%$ & \\
\hline \multicolumn{12}{|l|}{ ESTERS (cont) } \\
\hline isoamyl hexanoate & 5 & 25 & 10 & 29 & 70 & 29 & 20 & 20 & - & & *1218 \\
\hline methyl nonanoate & 5 & 12 & 10 & 3 & 5 & 4 & 5 & 11 & - & & *1226 \\
\hline isopropyl octanoate & 5 & 14 & - & & 50 & 19 & 15 & 9 & - & & *1235 \\
\hline isoamyle hexanoate & 20 & 23 & 10 & 26 & 170 & 21 & 105 & 29 & 320 & 5 & $* 1251$ \\
\hline amyl hexanoate & 10 & 21 & 110 & 8 & 20 & 21 & 10 & 28 & 10 & 4 & ${ }^{*} 1254$ \\
\hline 2-phenylethyl acetate & 15 & 17 & 20 & 8 & - & & 25 & 31 & 20 & 14 & *1263 \\
\hline methyl 4-decenoate & 10 & 5 & 35 & 11 & 30 & 19 & 35 & 23 & 30 & 16 & 1315 \\
\hline methyl decanoate & 145 & 28 & 855 & 10 & 635 & 15 & 290 & 29 & 250 & 4 & *1324 \\
\hline isopropyl nonanoate & - & & - & & 5 & 24 & 10 & 7 & - & & ${ }^{* 1} 330$ \\
\hline isobutyl octanoate & 10 & 13 & 25 & 6 & 30 & 26 & 15 & 20 & 30 & 3 & *1357 \\
\hline ethyl decanoate & 320 & 5 & 175 & 16 & 195 & 7 & 270 & 23 & 20 & 3 & *1397 \\
\hline 1-Me hexyl hexanoate & - & & - & & 60 & 26 & 10 & 20 & - & & 1405 \\
\hline 1-Me octyl butanoate & - & & 80 & 22 & 180 & 18 & 15 & 5 & - & & 1409 \\
\hline ester $\mathrm{C}_{5}$ octanoate & 10 & 3 & - & & 70 & 20 & 20 & 37 & - & & 1412 \\
\hline methyl undecanoate & 5 & 27 & - & & - & & - & & - & & 1424 \\
\hline isopropyl decanoate & 15 & 5 & 15 & 14 & 170 & 14 & 100 & 20 & - & & *1 434 \\
\hline 2-phenylethyl butanoate & - & & - & & - & & - & & 110 & 2 & ${ }^{* 1} 440$ \\
\hline isoamyl octanoate & 35 & 12 & 180 & 24 & 165 & 25 & 115 & 27 & 220 & $\overline{6}$ & 1449 \\
\hline amyl octanoate & - & & - & & - & & 10 & 23 & 10 & 4 & 1454 \\
\hline propyl decanoate & - & & - & & 15 & 31 & - & & - & & ${ }^{*} 1495$ \\
\hline methyl dodecanoate & 30 & 19 & 160 & 23 & 120 & 14 & 190 & 36 & 200 & 4 & ${ }^{*} 1527$ \\
\hline isobutyl decanoate & 20 & 9 & 75 & 3 & 55 & 11 & 40 & 32 & 60 & 8 & ${ }^{*} 1548$ \\
\hline 1-Me octyl hexanoate & 15 & 24 & - & & 10 & 5 & - & & - & & 1588 \\
\hline ethyl dodecanoate & 140 & 18 & 70 & 25 & 135 & 19 & 255 & 22 & - & & *1 595 \\
\hline 1-Me decyl butanoate & - & & - & & 20 & 8 & - & & - & & 1600 \\
\hline act-amyl decanoate & 10 & 27 & 5 & 25 & 120 & 15 & 70 & 32 & - & & 1608 \\
\hline isopropyl dodecanoate & 5 & 27 & - & & 20 & 8 & 45 & 30 & - & & ${ }^{*} 1622$ \\
\hline isoamyl decanoate & 55 & 7 & 370 & 4 & 250 & 17 & 280 & 37 & 550 & 12 & ${ }^{*} 1647$ \\
\hline amyl decanoate & 一 & & - & & - & & 40 & 38 & - & & \\
\hline
\end{tabular}


Blue cheeses

\begin{tabular}{|c|c|c|c|c|c|c|c|c|c|c|c|}
\hline \multirow{3}{*}{ Compounds } & & & & & & & & & & & \\
\hline & \multicolumn{2}{|c|}{$P F$} & \multicolumn{2}{|c|}{$P O$} & \multicolumn{2}{|c|}{$P G$} & \multicolumn{2}{|c|}{$C A U$} & \multicolumn{2}{|c|}{$A \cup V$} & \multirow[b]{2}{*}{$I R^{c}$} \\
\hline & $\mathrm{Ca}$ & $\%^{b}$ & C & $\%$ & $C$ & $\%$ & C & $\%$ & $C$ & $\%$ & \\
\hline \multicolumn{12}{|l|}{ ESTERS (cont) } \\
\hline 2-phenylethyl hexanoate & 10 & 11 & 55 & 13 & 30 & 12 & 100 & 28 & - & & ${ }^{\star} 1650$ \\
\hline methyl tetradecanoate & 25 & 12 & 80 & 10 & 40 & 9 & 110 & 38 & 130 & 9 & *1 728 \\
\hline isobutyl dodecanoate & 10 & 21 & - & & 10 & 4 & 20 & 32 & 30 & 18 & *1750 \\
\hline ethyl tetradecanoate & 95 & 35 & 70 & 43 & - & & 150 & 27 & 150 & 17 & *1798 \\
\hline ester $\mathrm{C}_{5}$ dodecanoate & - & & - & & 45 & 2 & - & & - & & 1805 \\
\hline isopropyl tetradecanoate & - & & - & & 15 & 10 & 40 & 14 & - & & 1828 \\
\hline isoamyl dodecanoate & - & & - & & 55 & 5 & 170 & 30 & - & & 1847 \\
\hline methyl hexadecanoate & 5 & 3 & 40 & 30 & 20 & 32 & 15 & 62 & - & & *1900 \\
\hline Total concentration & 1390 & & 2985 & & 3835 & & 3155 & & 2950 & & \\
\hline \multicolumn{12}{|l|}{ LACTONES } \\
\hline$\gamma$-hexalactone & - & & - & & 5 & 26 & - & & - & & 1083 \\
\hline$\gamma$-octalactone & - & & - & & 30 & 36 & 5 & 25 & - & & 1264 \\
\hline$\gamma$-decalactone & 10 & 9 & - & & 20 & 6 & 20 & 27 & 40 & 12 & *1474 \\
\hline$\gamma$-dodecenolactone & 10 & 37 & 45 & 7 & 55 & 15 & 30 & 25 & 150 & 12 & 1664 \\
\hline$\gamma$-dodecalactone & 30 & 22 & 210 & 11 & 215 & 9 & 370 & 28 & 1030 & 1 & ${ }^{*} 1690$ \\
\hline$\delta$-dodecalactone & - & & - & & - & & - & & 700 & 15 & ${ }^{*} 1740$ \\
\hline$\delta$-tetradecalactone & - & & - & & - & & - & & 310 & 1 & *1940 \\
\hline Total concentration & 50 & & 255 & & 325 & & 425 & & 2230 & & \\
\hline \multicolumn{12}{|l|}{ ALDEHYDES } \\
\hline 3-methyl butanal & - & & - & & $\operatorname{tr}$ & & - & & 160 & 31 & *633 \\
\hline hexanal & - & & - & & - & & - & & 10 & 33 & *830 \\
\hline benzaldehyde & 5 & 13 & 10 & 13 & 15 & 14 & - & & - & & *968 \\
\hline phenyl acetaldehyde & - & & - & & - & & - & & 60 & 7 & *1 070 \\
\hline decanal & - & & - & & - & & - & & 10 & 15 & *1225 \\
\hline 2,4-decadienal & - & & - & & - & & - & & 10 & 20 & 1320 \\
\hline Total concentration & 5 & & 10 & & 15 & & 0 & & 250 & & \\
\hline
\end{tabular}




\begin{tabular}{|c|c|c|c|c|c|c|c|c|c|c|c|}
\hline \multirow{3}{*}{ Compounds } & \multicolumn{10}{|c|}{ Blue cheeses } & \multirow[b]{3}{*}{$I R^{c}$} \\
\hline & \multicolumn{2}{|c|}{$P F$} & \multicolumn{2}{|c|}{$P O$} & \multicolumn{2}{|c|}{$P G$} & \multicolumn{2}{|c|}{$C A U$} & \multicolumn{2}{|c|}{$A \cup V$} & \\
\hline & $\mathrm{Ca}^{a}$ & $\%^{b}$ & $c$ & $\%$ & $c$ & $\%$ & $c$ & $\%$ & C & $\%$ & \\
\hline \multicolumn{12}{|l|}{ BENZENIC COMPOUNDS } \\
\hline methyl benzene & 10 & 14 & 10 & 28 & - & & - & & 30 & 30 & 768 \\
\hline dimethyl benzene & 5 & 11 & 5 & 14 & - & & - & & 10 & 5 & 856 \\
\hline 1,1-diMe decyl benzene & 10 & 47 & - & & - & & - & & - & & 1708 \\
\hline Total concentration & 25 & & 15 & & 0 & & 0 & & 40 & & \\
\hline \multicolumn{12}{|l|}{ ANISOLES } \\
\hline 4-methoxytoluene & 80 & 35 & 15 & 2 & 15 & 5 & 5 & 63 & 20 & 5 & *1 035 \\
\hline 1,3-dimethoxy benzene & 10 & 13 & 80 & 9 & - & & 10 & 26 & - & & ${ }^{*} 1164$ \\
\hline 3,4-dimethoxytoluene & 5 & 62 & - & & - & & - & & - & & *1243 \\
\hline Total concentration & 95 & & 95 & & 15 & & 15 & & 20 & & \\
\hline \multicolumn{12}{|l|}{ PYRAZINES } \\
\hline 2,6-dimethyl pyrazine & 20 & 24 & 30 & 19 & 25 & 32 & 10 & 2 & - & & *910 \\
\hline 2,3,5,-trimethyl pyrazine & - & & - & & - & & - & & 30 & 32 & *1 015 \\
\hline Total concentration & 20 & & 30 & & 25 & & 10 & & 30 & & \\
\hline \multicolumn{12}{|l|}{ MISCELLANEOUS } \\
\hline benzothiazole & $\overline{5}$ & 9 & - & & - & & - & & - & 2 & 1241 \\
\hline indole & 5 & 3 & 5 & 15 & 5 & 15 & 一 & & - & & *1308 \\
\hline Total concentration & 10 & & 5 & & 5 & & 0 & & 80 & & \\
\hline
\end{tabular}




\begin{tabular}{|c|c|c|c|c|c|c|c|c|c|c|c|}
\hline \multirow{3}{*}{ Compounds } & \multicolumn{10}{|c|}{ Blue cheeses } & \multirow[b]{3}{*}{$I R^{c}$} \\
\hline & \multicolumn{2}{|c|}{$P F$} & \multicolumn{2}{|c|}{$P O$} & \multicolumn{2}{|c|}{$P G$} & \multicolumn{2}{|c|}{$C A U$} & \multicolumn{2}{|c|}{$A U V$} & \\
\hline & $C^{a}$ & $\%^{b}$ & $C$ & $\%$ & $c$ & $\%$ & $c$ & $\%$ & $c$ & $\%$ & \\
\hline \multicolumn{12}{|c|}{ UNIDENTIFIED COMPOUNDS } \\
\hline Oxygenated sesquiterpene & 70 & 9 & - & & - & \multirow{8}{*}{2} & - & & - & & 1410 \\
\hline Sesquiterpene & 15 & 7 & 20 & 15 & - & & - & & - & & 1446 \\
\hline Sesquiterpene & - & & - & & 20 & & - & & - & & 1452 \\
\hline Sesquiterpene & 15 & 25 & 30 & 22 & - & & - & & - & & 1455 \\
\hline Sesquiterpene & 25 & 26 & 10 & 12 & - & & - & & - & & 1512 \\
\hline Oxygenated sesquiterpene & 25 & 24 & 15 & 40 & - & & - & & - & & 1517 \\
\hline Sesquiterpene & 5 & 7 & - & & - & & - & & - & & 1532 \\
\hline Sesquiterpene & 5 & 0 & - & & - & & - & & - & & 1553 \\
\hline Total concentration & 5195 & & 5980 & & 20 & & 3925 & & 0 & & \\
\hline Total & 21950 & & 27040 & & 46980 & & 20670 & & 23500 & & \\
\hline
\end{tabular}


were always more important than variations between replicates.

The sum of all quantified compounds (last line of table IV) gave an evaluation of the total odorous profile. By applying a correcting coefficient to the main chemical classes (1.43 for methyl-ketones which corresponded to an average recovery of $70 \%, 1.66$ for alcohols and lactones which corresponded to a $60 \%$ recovery), a more accurate evaluation of the total odorous profile, ie 29.4, 35.6 and $67.3 \mathrm{mg} / \mathrm{kg}$, respectively for the Roquefort samples PF, $P O$ and $P G, 27.5 \mathrm{mg} / \mathrm{kg}$ for the Bleu des Causses and $34.7 \mathrm{mg} / \mathrm{kg}$ for the Bleu d'Auvergne, was obtained. These figures should be considered cautiously since all correcting coefficients could not be calculated, but they allowed a global comparison of the samples. Thus, odorous volatile compounds were more abundant in the three Roquefort cheeses than in the Bleu des Causses sample, the PG cheese being twice as rich as the two other samples. The Bleu d'Auvergne sample was quantitatively intermediate between the Roquefort samples $P O$ and $P G$.

The predominant components of blue cheese aroma, methyl-ketones and related secondary alcohols, originate from fatty acids (Kinsella, Hwang, 1976). So we tried to relate the total odorous profile (last line of table IV) with the concentration of FFA (table II). An obvious correlation was observed for the Roquefort and the Bleu d'Auvergne samples: both FFA and volatile compounds were twice as abundant in Roquefort PO, PF and Bleud 'Auvergne, and these 3 samples contained similar concentrations of both FFA and volatile compounds. However, despite its higher content in FFA, the Bleu des Causses did not show the richest odorous profile (and especially the highest content in methyl ketones and secondary alcohols). This could be consistent with the observations of Kin- sella and Hwang (1976): the rate of release of FFA could be a limiting factor in methyl-ketone production since FFA are toxic to the mycellium of Penicillium roqueforti (particularly long-chain fatty acids) and inhibit methyl-ketone formation. However, not only lipolysis rate can affect the odorous profiles. Many other properties of the moud, not measured here, influence the production of aroma compounds in blue cheese (proteolytic activity, growth rate).

\section{Ketones}

Methyl-ketones represented about half of the odorous profile for all samples, and accounted for $75 \%$ of the odorous profile for Roquefort PG. 2-heptanone and 2nonanone were the most abundant ketones except for the Bleu des Causses, in which 2-pentanone was predominant and represented $16.6 \%$ of the total odorous profile. This ketone was also very abundant in Roquefort PG $20 \%$ of the odorous profile). 2-undecanone was important in the Bleu d'Auvergne profile: its concentration was similar to that of 2-heptanone.

Roquefort PF was considered to have a neutral flavour and contained the lowest concentrations of both methyl-ketones and FFA. On the other hand, PG was often judged as good, but a little too pungent or "burning", which could be explained by the abundance of both methyl-ketones and FFA. These results confirmed the general idea that the sensory quality of blue cheese is correlated with these two classes of chemicals. However, the methylketones and FFA content of the Roquefort $\mathrm{PO}$ en FF were similar, and only PO was judged to possess the ideal flavour of Roquefort, which indicates the importance of other odorous compounds in the typical flavour of Roquefort.

The quantitative data obtained in this word were compared to the literature data 
(table V). All of them had been obtained by derivatization of monocarbonyl compounds to dinitrophenylhydrazones, first described by Schwartz, Parks (1963). Our figures were generally 2 to 10 -fold lower than all other results. The concentrations found in Roquefort PG (the highest concentrations in this work) were similar to those obtained by Anderson, Day (1966) for an imported Roquefort sample. However, this cheese was, for them, the poorest sample. The variability of samples of various origins may explain these differences.

A few unsaturated ketones were detected here. Only 8-nonen 2-one had been reported in Normanna cheese (Svensen, Ottestad, 1969). The concentrations were again more important in their work : up to $24 \mathrm{mg} / \mathrm{kg}$ instead of $1 \mathrm{mg} / \mathrm{kg}$ (maximum value found here in Bleu d'Auvergne cheese). Karahadian et al (1985), who found this coumpound in Brie cheese, proposed a mechanism for its formation involving the "classical" oxidative decarboxylation of 9-decenoic acid (emanating from the lipoxygenase-mediated cleavage of polyunsaturated fatty acids).

Low amounts of acetophenone occurred in Roquefort and Bleu des Causses. This compound, which has been found in some washed-rind type cheeses (Adda, Dumont, 1974; Parliment et al, 1982) and in Comté cheeses (Dumont et al, 1981), where it could be a metabolite of the smear microflora, was reported for the first time in blue cheese.

\section{Alcohols}

Alcohols represented $15-20 \%$ of the total odorous profile of Roquefort and Bleu des Causses and more than a third for the Bleu

Table V. Methyl-ketones content of blue cheeses of different origin (minimum and maximum value found in each work). A : Normanna cheese (Svensen \& Ottestad, 1969) - 22 samples; B : Roquefort cheese (Schwartz et al, 1963) - 3 samples; C : Roquefort cheese (Anderson \& Day, 1966) - 2 samples; D : American Blue cheese (Anderson \& Day, 1966) - 5 samples; E : real concentrations obtained in our work, calculated with a correcting factor of 1.43 (corresponding to a $70 \%$ recovery of the methyl ketones).

Quantités de méthyl-cétones trouvées dans différents bleus : données bibliographiques (valeurs minimales et maximales citées dans chaque travail). A : fromage Normanna (Svensen, Ottestad, 1969) 22 échantillons; B : Roquefort (Schwartz et al, 1963) - 3 échantillons; $C$ : Roquefort (Anderson, Day, 1966) - 2 échantillons; $D$ : Bleu américain (Anderson, Day, 1966) - 5 échantillons; $E$ : concentrations réelles obtenues dans notre travail, calculées avec un coefficient de correction de 1,43 (ce qui correspond à un pourcentage de récupération des méthyl cétones de $70 \%$ ).

$m g / k g$ blue cheese

Chain length
$A$
$B$
C
$D$
E

\begin{tabular}{rrrrrr}
\hline $\mathrm{C}_{5}$ & $1.3-142.0$ & $3.6-20.9$ & $6.5-20.9$ & $3.6-19.2$ & $0.5-13.5$ \\
$\mathrm{C}_{7}$ & $4.8-100.0$ & $17.6-71.0$ & $17.9-71.8$ & $17.6-69.9$ & $2.5-18.0$ \\
$\mathrm{C}_{9}$ & $3.9-145.0$ & $19.8-88.3$ & $19.8-88.3$ & $13.9-78.9$ & $2.3-14.3$ \\
$\mathrm{C}_{11}$ & $\mathrm{tr}-20.3$ & $2.4-29.9$ & $4.9-29.9$ & $2.4-6.7$ & $0.7-2.1$ \\
\hline
\end{tabular}


d'Auvergne sample. Secondary alcohols were generally more abundant than primary alcohols, except for Bleu d'Auvergne, for which primary alcohols were twice as abundant.

Odd-numbered secondary alcohols were found in all cheeses. Conversion rates of methyl-ketones to the corresponding alcohols, vary according to the strain and chain length of that strain (Kinsella, Hwang, 1976). However, these rates were similar for Bleu des Causses and Roquefort $P G$ which had been manufactured with the same strain $(25,30$ and $10 \%$, respectively, for 2-heptanone, 2-nonanone and 2undecanone).

Two papers reported a quantitative analysis of secondary alcohols in blue cheese : the concentrations found in our work for 2-pentanol, 2-heptanol and 2nonanal were similar to those found by Anderson, Day (1966) in American Blue and Roquefort samples. Concentrations obtained by Svensen, Ottestad (1969), for 22 samples of Normanna cheese were, however, 1 to 3 -fold higher.

Even-numbered secondary alcohols were also present at very low levels. They probably did not contribute to the blue cheese aroma.

Several primary alcohols found here had been already reported in blue cheeses by Day and Anderson (1965) and Ney and Wirotama (1972), without any quantitative indication. They may impart a fruity, nutty not to the flavour of cheese (Kinsella, Hwang, 1976). 3-methyl 1-butanol, which occurred in both Roquefort PF and PG, was particularly abundant in the Bleu d'Auvergne cheese, where it represented almost $40 \%$ of the alcohol class. 1pentanol was found only in Bleu des Causses. Phenyl-ethanol was found in all cheeses except Roquefort PG, and was 10-fold more abundant in Bleu d'Auvergne (its real concentration in this cheese was 2 $\mathrm{mg} / \mathrm{kg}$ ). With its reminiscent odour of yeast, even at low levels, it contributes to the background flavour and to the typical aroma of blue cheese (Anderson, Day, 1966).

Low amounts of unsaturated alcohols were detected in blue cheeses. Originating from the oxidation of polyunsaturated fatty acids by $P$ roqueforti, these compounds have been often reported in Brie or Camembert cheeses (Karahadian et al, 1985), where they contributed largely to the aroma. 1-octen 3-ol is also desirable for blue cheese aroma at about 5 to 50 ppm (Moinas et al, 1974; Ney et al, 1975), but this concentration was not reached in the samples analyzed.

\section{Phenols}

The 3 phenolic compounds: 2-methyl 5ethyl phenol, 4-ethyl phenol and 3-propyl phenol, which occurred in Roquefort samples, have not, as far as we know, been reported before in any kind of cheese. They probably originated from the microbiological breakdown of an amino-acid. Such a mechanism was demonstrated for phenol, one of the major flavour compounds of the surface-ripened cheeses (Parliment et al, 1982), which is formed during tyrosine degradation. Incidentally, despite their low thresholds, they were found at such low levels that their contribution to the aroma must be very marginal.

\section{Esters}

The most important chemical class for the number of compounds was the ester class. Fourteen of the 57 esters found here were detected by Day and Anderson (1965); the others are reported for the first time in blue cheese. They represented $6-15 \%$ of the total odorous profile (15\% in Bleu des Causses, which contained 49 esters). 
Some of them were found at rather high levels: methyl-decanoate represented $3.2 \%$ of the total odorous profile for Roquefort PO, and $1.4 \%$ for both Roquefort PG and Bleu des Causses. Isoamyldecanoate represented, respectively, 1.4\% and $2.3 \%$ of the total odorous profile for Roquefort $\mathrm{PO}$ and Bleu d'Auvergne, and ethyl-decanoate, $1.5 \%$ of the Roquefort PF profile. In addition, Bleu d'Auvergne contained heptyl-acetate and 2-phenyl-ethyl butanoate, which were not found in the other samples.

Whether ester formation results from a chemical equilibrium or an enzymic mechanism has not yet been elucidated, but esterification is known to be a secondary reaction between primary volatile compounds. The ester content of cheeses has been shown to increase during ripening (Dumont, Adda, 1978). However, despite a ripening period which was twice as long for Roquefort PG than for Bleu des Causses, their ester contents were quantitatively similar.

Esters possess fruity notes and may contribute to the aroma by minimizing the sharpness and bitterness imparted by fatty acids and animes, respectively. This result was obtained by Anderson, Day (1966) with an ethyl-butanoate, methyl-hexanoate and octanoate addition, which reduced the harshness of a synthetic mixture containing methyl-ketones, secondary alcohols and fatty acids. Ethyl-esters of butanoic, hexanoic and octanoic acid were incorporated in a blue cheese flavor patented by Ney et al (1975). The flavour specificity of the 3 types of blue cheese studied here could partially originate from the both qualitative and quantitative differences in their ester content. This could, in particular, explain the character of Roquefort PO with regard to the neutral flavour of Roquefort PF; all chemical classes were quantitative- ly similar, except esters, of which Roquefort $\mathrm{PO}$ contained twice as much as Roquefort PF.

\section{Lactones}

Lactones were found only at low concentrations in Roquefort and Bleu des Causses, but they represented about $10 \%$ of the total odorous profile of Bleu d'Auvergne. The higher content of Bleu d'Auvergne could be related to the use of pasteurized milk, since the heating of milk products has been shown to increase their lactone level.

Both $\gamma$ - and $\delta$-lactones were found in our samples, $\gamma$-dodecalactone being predominant. Blue cheeses have already been shown to contain both $\delta$ - and $\gamma$-lactones (Jolly, Kosikowski, 1975). But $\delta$-dodecalactone, and above all $\delta$-tetradecalactone, were, in that study, the major lactones formed. Jolly, Kosikowsky (1975) noticed a decrease of $\delta$-lactones during ripening. This phenomenon, also observed by Wong et al (1975) in Cheddar cheese, could explain, in our work, the absence of $\delta$ lactones in Roquefort and Bleu des Causses, which had a longer ripening period than Bleu d'Auvergne.

Lactones in cheese are supposed to be formed by hydrolysis of the hydroxy-fatty acids (a normal constituent of milk fat) followed by lactonization (Jolly, Kosikowski, 1975). Other mechanisms have been proposed (Adda et al, 1982), but none of them have been proved. The differences in lactone content are therefore directly related to the lactone potential of milk. The amount of lactone precursors, greater for cow's milk than for ewe's milk (Dimick et al, 1969 ), has been shown to be influenced by many different factors such as feed, season, breed, stage of lactation (Dimick, Harner, 1968) or diet of animals (Urbach, 1982). 
The lactone concentrations found here were lower than those reported by Jolly, Kosikowski (1975): up to $18 \mathrm{mg} / \mathrm{kg}$ of cheese. $\gamma$-dodecalactone, which has a detection threshold of about $1 \mathrm{ppm}$ in butter (according to Siek et al, 1969), may be important for the background flavour of Bleu d'Auvergne, and perhaps for that of Bleu des Causses and Roquefort PG, if the possible synergic effect of lactones is taken into account. However, the contribution of both $\delta$-dodecalactone and $\delta$-tetradecalactone, which occurred in Bleu d'Auvergne at levels lower than their detection threshold (which were $5.4 \mathrm{ppm}$ in a lactone mixture), is debatable.

\section{Other compounds}

A few aldehydes were found in Bleu d'Auvergne. Some of them had been already reported in blue Cheese (3-methyl butanal, phenyl-acetaldehyde: Day, Anderson, 1965; Ney, Wirotama, 1972). Only benzaldehyde was detected in Roquefort. No aldehyde was found in Bleu des Causses. The importance of aldehydes in the flavour of blue cheese has not yet been elucidated. Probably formed by degradation of amino-acids (Kinsella, Hwang, 1976), they may impart an "unclean", harsh and dull flavour (as observed in Cheddar for phenyl acetaldehyde and 3-methyl butanal, by Dunn, Lindsay, 1985).

Several anisole compounds were found: 1,3-dimethoxybenzene, 4-methoxy- and 3,4-dimethoxytoluene. They contribute to the aroma of Roquefort $\mathrm{PO}$ and $\mathrm{PF}$ with their, respectively, hazelnut-like, floral or sweet notes. Already found in Camembert (Dumont et al, 1976), they may originate from the breakdown of lignin occurring in feed.

\section{Undentified compounds}

A few presumed sesquiterpenic compounds were found in Roquefort PO and PF. They may have been transferred from the forage to the milk without degradation (Dumont, Adda, 1978). Because they occurred at very low levels and did not exhibit any odor at the sniffing-port of the chromatograph, their contribution to the aroma was negligible.

\section{CONCLUSIONS}

Besides carbonyl compounds, always reported as being responsible for the typical blue cheese flavor, many other compounds were found in the odorous profile of all cheeses. Sulfur compounds, esters, anisoles, more numerous in Roquefort and Bleu des Causses, lactones and aldehydes, more abundant in Bleu d'Auvergne, possibly contributed to the aroma and may partly explain the specificity of each cheese. However, other compounds (such as fatty acids and their soaps, amino acids, amines) play a major role in both flavour and taste of blue cheese. The quantification of these compounds could provide further information on blue cheese flavor.

\section{ACKNOWLEDGMENTS}

We are grateful to M. Assenat (Laboratoire du Groupe Société des Caves de Roquefort) for supplying the cheese samples.

\section{REFERENCES}

Adda J, Dumont JP (1974) Les substances responsables de l'arôme des fromages à pâte molle. Lait 54, 1-21 
Adda J, Gripon JC, Vassal L (1982) The chemistry of flavour and texture generation in cheese. Food Chem 9, 115-129

Anderson DF (1966) Flavor chemistry of blue cheese. Diss Abstr 26, 6636-6637

Anderson DF \& Day EA (1966) Quantification, evaluation and effect of certain microorganisms on flavor components of blue cheese. $J$ Agric Food Chem 14, 241-245

Day EA (1967) Cheese flavor. In : The Chemistry and Physiology of Flavours (HW Schultz, EA Day, LM Libbey, eds), The AVI Publishing Company Inc, Westport, 331-361

Day EA \& Anderson DF (1965) Gas chromatographic and mass spectral identification of natural components of the aroma fraction of blue cheese. J Agric Food Chem 13, 2-4

Dimick PS \& Harner JL (1968) Effect of environmental factors on lactone potential in bovine milk fat. J Dairy Sci 51, 22-27

Dimick PS, Walker NJ \& Patton S (1969) Occurence and biochemical origin of aliphatic lactones in milk fat: a review. J Agric Food Chem 17, 649-655

Dumont JP, Adda J (1978) Occurrence of sesquiterpenes in mountain cheese volatiles. $J$ Agric Food Chem 26, 364-367

Dumont JP, Roger S, Adda J (1976) L'arôme du Camembert : autres composés mineurs mis en évidence. Lait 56, 595-599

Dumont JP, Adda J, Rousseaux P (1981) Exemple de variation de l'arôme à l'intérieur d'un même type de fromage : le Comté. Lebens Wiss Technol 14, 198-202

Dunn HC, Lindsay RC (1985) Evaluation of the role of microbial Strecker-derived aroma compounds in unclean-type flavors of Cheddar cheese. J Dairy Sci 68, 2859-2874

Forss DA, Holloway GL (1967) Recovery of volatile compounds from butter oil. $J$ Am Oil Chem Soc 44, 572-575

Jolly RC, Kosikowski FV (1975) Quantification of lactones in ripening pasteurized milk blue cheese containing added microbial lipases. $J$ Agric Food Chem 23, 1175-1176

Karahadian C, Josephson DB, Lindsay RC (1985) Contribution of Penicillium $s p$ to the flavors of Brie and Camembert cheese. I Dairy Sci 68, 1865-1877

Kinsella JE, Hwang DH (1976) Enzymes of Penicillium roqueforti involved in the biosynthesis of cheese flavor. Crit Rev Food Sci Nutr 191-228
Madkor S, Fox PF, Shalabi SI, Metwalli NH (1987) Studies on the ripening of Stilton cheese: lipolysis. Food Chem 25, 93-109

Moinas M, Groux MJA, Hormann I (1974) Aroma compositions for imparting a Camembert of blue cheese flavor to foods. Swiss Patent CH 552949

Ney KH, Wirotama IPG (1972) Investigation of the flavour of "Edelpilzkäse", a German blue mould cheese. Z Lebensm Unters Forsch $149,275-279$

Ney KH, Wirotama IPG, Freytag WG (1975) Blue cheese flavor. British Patent 1381737

Parliment TH, Kolor MG, Rizzo DJ (1982) Volatile components of Limburger cheese. J Agric Food Chem 30, 1006-1008

Qvist IH, Von Sydow ECF (1976) Unconventional proteins as aroma precursors. Chemical analysis of the volatile compounds in unheated and heated rapeseed protein model systems. J Agric Food Chem 24, 437-442

Schwartz DP, Parks OW (1963) Quantitative analysis of methyl ketones in blue cheese fat. $\checkmark$ Dairy Sci 46, 989-990

Schwartz DP, Parks OW, Boyd EN (1963) Methyl ketones in Roquefort cheese. I Dairy $\mathrm{Scl}$ $46,1422-1423$

Shankaranarayana ML, Raghavan B, Abraham KO, Natarajan CP (1974) Volatile sulfur compounds in food flavours. Crit Rev Food Technol 4 (3), 395-435

Siek TJ, Albin IA, Sather LA, Lindsay RC (1969) Taste thresholds of butter volatiles in deodorized butteroil medium. J Food Sci 34, 265267

Stark W, Urbach G, Hamilton JS, Forss DA (1973) Volatile compounds in butter oil. III. Recovery of added fatty acids and $\delta$-lactones from volatile-free butter oil by cold-finger molecular distillation. J Dairy Res 40, 39-46

Svensen A, Ottestad E (1969) Qualitative and quantitative analysis of flavour components in Normanna cheese. Dairy Res Inst Agric College of Norway 3-4, 3-15

Urbach $G$ (1982) The effect of different feeds on the lactone and methyl-ketone precursors of milk fat. Lebensm Wiss Technol 15, 62-67

Wong NP, Ellis R, La Croix DE (1975) Quantitative determination of lactones in Cheddar cheese. J Dairy Sci 58, 1437-1441

Woo AH, Kollodge S, Lindsay RC (1984) Quantification of major free fatty acids in several cheese varieties. J Dairy Sci 67, 874-878 